\title{
CIRCADIAN ACTIVITY RHYTHM OF THE HOUSE FLY CONTINUES AFTER OPTIC TRACT SEVERANCE AND LOBECTOMY
}

\author{
Charlotte Helfrich*, Bronislaw Cymborowski† and Wolfgang Engelmann* \\ *Institute für Biologie I, University of Tübingen, D7400 Tübingen', FRG \\ +Department of Invertebrate Physiology, Zoological Institute, University of Warsaw, Warszawa, Poland
}

(First received in October 1984; accepted in revised form in February 1985)

\begin{abstract}
Under constant conditions, locomotor activity in about $50 \%$ of 63 adult Musca domestica continued to be rhythmic after bilateral severance of optic tracts or bilateral lobectomy. Apparently, the optic lobes of Musca do not contain the oscillator for rhythmic control of locomotor activity as has been proposed for other insects. In $20 \%$ of the individuals, several circadian components of activity rhythms were found after operation indicating a role of the optic lobes in the coupling of oscillators. The remaining $30 \%$ of the flies with severed optic tracts appeared to be arrhythmic. Most of these flies had vacuolized tissue in the central brain. However, disruption of rhythmicity did not correlate with a common pattern of degeneration. Therefore no conclusions can be drawn as to the localization of the circadian control of locomotor activity in the brain. Flies showing an arrhythmic activity pattern could still be synchronized by LD cycles. Activity did not occur solely during the light period as is the case in controls; but was phase delayed by about $6 \mathrm{hr}$ towards the dark period. Since all flies with severed optic tracts could be synchronized by LD cycles, Musca domestica must possess extraocular photoreceptors.
\end{abstract}

Key words-Activity, circadian rhythm, Musca domestica, optic lobe, insect brain, photoreceptor.

\section{Introduction}

Quite a number of studies of the physiology of insect circadian rhythms have focused on its anatomical localization $(1,2)$. NishijtsutsujiUo and Pittendrigh (3) presented evidence for the involvement of optic lobes of cockroaches (Periplaneta americana and Leucophaea made$\mathrm{rae}$ ) in regulating the locomotor activity rhythm. Thus, bilateral ablation of the optic lobes or sectioning of the optic tracts caused arrhythmicity. The same operation, performed unilaterally, did not abolish the rhythms. These findings have been confirmed (4-6) and extended to other insect species (Teleogryllus commodus: 7, Gryllus bimaculatus: 8, Carabus problematicus: 9). The region within the optic lobes controlling the activity rhythm seems to be the lobula in the case of cockroaches (1) and the lamina/medulla region in the case of Gryllus (8). In the cockroach Thythmic activity depends on the structural connections between optic lobes: 4-8 wk after implantation new neural connections between optic lobes and midbrain were established and the rhythm restored. The period length was imposed by the donor lobe (6). Regeneration of neural connections restores rhythmicity also in Gryllus (8).

Results of parabiosis experiments in cockroaches (Periplaneta americana) and Acheta domestica (10) indicate, however, that the locomotor activity rhythm can be driven via a hormonal pathway. Apparently the brain can release a diffusible factor capable of driving circadian activity rhythms. At least in Acheta domestica $(11,12)$ and in Drosophila melanogaster $(13,14)$ this factor seems to be produced rhythmically by neurosecretory cells of the brain. In silkmoths both eclosion and flight activity are rhythmic and controlled by a circadian pacemaker in the cerebral lobes $(15,16)$ : ablation of the optic lobes did not disrupt rhythmicity.

Optic lobes seem to be dispensable for the expression oi rhythmic locomotor activity in

${ }_{+}$Author to whom reprint requests should be addressed.

Abbreviations: LL, continuous illumination with white light, RR, continuous red light; LD, light dark cycle; a activity

time; $\varrho$, rest time; $\mathbf{a}: \boldsymbol{\rho}$, proportion of activity to rest time; $\boldsymbol{\tau}$. period length; min, minute (s); d. dav(s): wk. week (s). 
Drosophila (17), in the mosquito Culex pipiens (18), and, as shown in the following, in house flies.

\section{Materials and Methods}

Experiments were performed on the house fly Musca domestica. The insects were reared in LD $16: 8$ at $25^{\circ} \mathrm{C}$. The adult flies taken for the experiments were not older than $10 \mathrm{~d}$.

Locomotor activity was monitored separately in each fly under RR at $20 \pm 1^{\circ} \mathrm{C}$ (19). Recordings were carried out for about $10 \mathrm{~d}$ before treatment. All arrhythmic animals were discarded; only those with clear circadian activity rhythms were used as experimental or control animals.

Experimental groups consisted of insects in which either both optic tracts were severed or both optic lobes were removed at different circadian times. During the operation which lasted usually 5-10 min, insects were exposed to cold fiber glass light of $6 \times 10^{4} \mathrm{~lx}$. Controls were either sham operated or received illumination alone.

In order to transect the optic tracts the insect was mounted in soft plasticine and illuminated. The head was bent forward to facilitate access to the optic tracts through the rear part of the head capsule. The cuts were performed with a fine scalpel using two cuticular landmarks on the surface of the rear head capsule (Figure 1). Lobectomy was performed after previously severing the optic tracts. Following this, the optic lobes were separated from the eye cup with a needle and then removed using fine forceps. In control flies all steps of operation were performed prior to severing the optic tracts. The wounds were sealed with wax (melting point $30^{\circ} \mathrm{C}$ ).

After surgical treatment the activity of the insect was monitored for about two wk under RR and in some cases for a further wk under LD 12 : 12 conditions. In order to see whether blind, but unoperated flies can be synchronized by a LD cycle, the complex eyes of some flies were covered with black wax and illuminated with different intensities.

Activity records were analysed using different methods of time series analysis such as

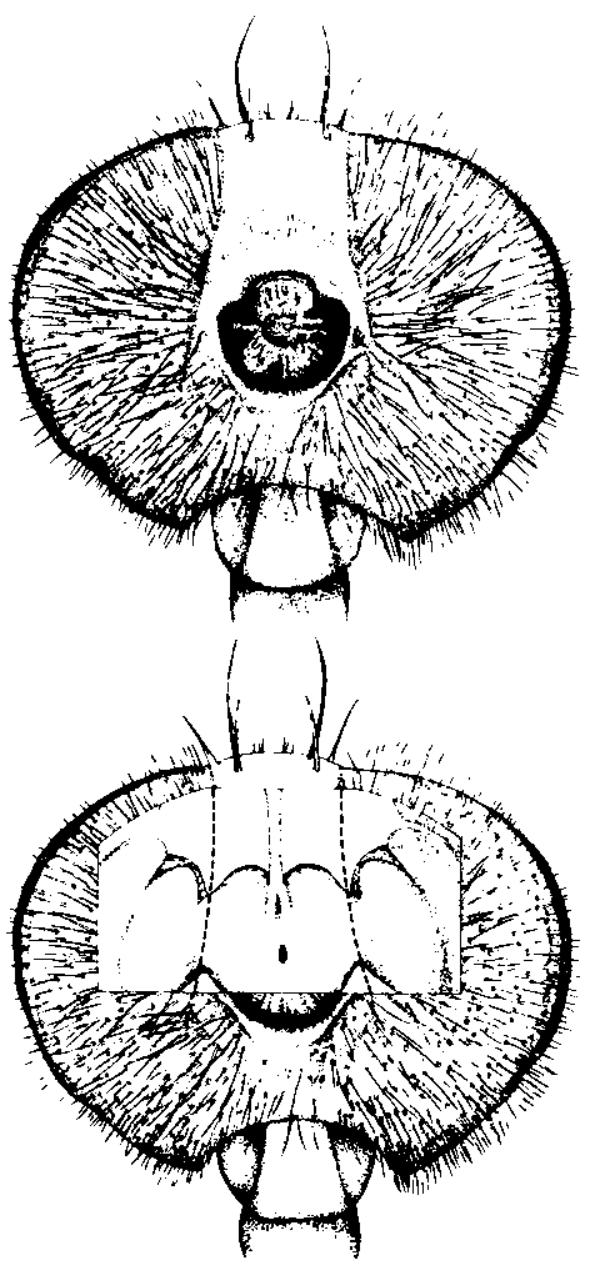

Figure 1. Caudal view of head of adult Musca domestica. (a) To severe the optic tracts, a landmark between the central and peripheral area of the dorsal part was used. (b) The position of the brain and the path of the cut is shown through a window in the lower drawing. Modified after Straussfeld (36).

periodogram, signal average, and complex demodulation (20). Activity levels were determined from the Esterline Angus recordings by assigning an activity score to each 20 min interval in units ranging from 0 (no activity) to 4 (hyperactive). To test the significance of the results, nonparametric tests such as $\chi^{2}$ test. Mann-Whitney- $U$-test, Wilcoxon test and Spearman rank correlation test were used (2l).

The position of optic tract severance was determined by histological examination. After 
postoperative locomotor activity the head was cut off and mounted upside down on a paraffin block. The caudal wall of the head capsule was removed taking care not to destroy any major nerve connections. The exposed brain was stained in situ for $10 \mathrm{~min}$ with Chicago blue dissolved in insect saline (22). The stained brain was rinsed with fresh saline and then fixed for 3 hr in Carnoy fluid. After dehydration the head was embedded in paraffin, sectioned $(10 \mu \mathrm{m})$, cleared with xylene and embedded in Entellan (Merck) prior to microscopical examination.

\section{Results}

(1) Characteristics of locomotor activity rhythm In order to characterize the locomotor activity rhythm of Musca domestica, flies were recorded under LD 12 : 12, under RR and LL (1.8 lx). Under all recording conditions no difference was found in the circadian behaviour of males and females. Under LD conditions activity was restricted to the light span, starting immediately after lights on and stopping immediately after lights off. The highest amount of activity occurred at the end of the light span. The majority of flies (85\%) showed unimodal activity patterns under LD and shortened their activity time when transferred to free running conditions. Some flies (15\%) showed a bimodal distribution with some activity after lights on and a more pronounced peak before lights went off. After transfer to $\mathrm{RR}$, only the second component persisted.

During the first few $d$ of RR $70 \%$ of the flies had a period length of about $23 \mathrm{hr}$, which then increased to about $25 \mathrm{hr}$ without further change. About $75 \%$ of all flies monitored showed clear free running thythms. The remaining $25 \%$ of the flies were either arrhythmic or had a complex rhythm with more than one component as revealed by periodogram analysis. Under LL conditions $(1.8 \mathrm{~lx}) 77 \%$ of the flies were hyperactive and appeared to be arrhythmic.

\section{(2) Effect of optic tract severance on activity rhythm}

Most of the flies with transected optic tracts had less defined activity rhythms after operation compared with the preoperative behaviour. This was due to increased mean activity levels (in $75 \%$ of all operated flies) and lack of clear rest time ( $\rho$ ) (Table 1). Changes were also observed in the activity patterns of operated flies. Three major types could be distinguished (Table 2): about half of the operated flies showed a clear circadian

Table 1. Activity levels, changes in activity level, ratio of actvity time ( a ) to rest time ( $\rho$ ) of Musca domestica before and after treatment. Mean values and standard errors. Number of cases in parentheses

\begin{tabular}{|c|c|c|c|}
\hline & $\begin{array}{c}\text { Activity } \\
\text { level }\end{array}$ & $\begin{array}{c}\text { Mean increase } \\
\text { of activity } \\
\text { level }\end{array}$ & $\mathbf{a}: \boldsymbol{e}$ \\
\hline Preoperative & $1.64 \pm 0.08(53)$ & \multirow[b]{2}{*}{$0.70 \pm 0.12 *(53)$} & $0.774 \pm 0.094(20)$ \\
\hline Postoperative & $2.31 \pm 0.10(53)$ & & $2.428 \pm 0.407(20)$ \\
\hline Before sham & $1.53 \pm 0.10(20)$ & \multirow{2}{*}{$0.09 \pm 0.16 \quad(20)$} & $0.708 \pm 0.096(20)$ \\
\hline After sham & $1.62 \pm 0.17(20)$ & & $0.721 \pm 0.060(20)$ \\
\hline $\begin{array}{l}\text { Before light } \\
\text { pulse }\end{array}$ & $1.61 \pm 0.16(15)$ & \multirow{2}{*}{$0.27 \pm 0.13$} & $0.727 \pm 0.045(15)$ \\
\hline $\begin{array}{l}\text { After light } \\
\text { pulse }\end{array}$ & $1.75 \pm 0.17(15)$ & & $0.940 \pm 0.148(15)$ \\
\hline
\end{tabular}

*Significant at 0.005 (Wilcoxon test). 
Table 2. Activity patterns of Musca domestica after bilateral severance of optic tracts, bilobectomy, sham operation and light pulse treatment only. Except for the first column all figures are percentages of each group (rows)

\begin{tabular}{lcccc}
\hline & $\begin{array}{c}\text { Number } \\
\text { of cases }\end{array}$ & $\begin{array}{c}\text { Rhythmic } \\
\text { pattern }\end{array}$ & $\begin{array}{c}\text { Complex } \\
\text { rhythmicity }\end{array}$ & $\begin{array}{c}\text { Arrhythmic } \\
\text { pattern }\end{array}$ \\
\hline $\begin{array}{l}\text { Optic tracts } \\
\text { severed }\end{array}$ & 53 & 52.8 & 18.9 & 28.3 \\
Lobectomy & 10 & 50.0 & 30.0 & 20.0 \\
Sham & 20 & 100.0 & 0.0 & 0.0 \\
Light pulse & 15 & 100.0 & 0.0 & 0.0 \\
\hline
\end{tabular}

rhythmicity after bilateral optic tract transection (example: Figure 2) or after lobectomy (see Figure 7a). About $30 \%$ of the insects with both optic tracts severed and $20 \%$ of the lobectomized flies were arrhythmic (example see Figure $7 \mathrm{c}$ ). Complex rhythmicity was found in $20 \%$ of the flies with transected optic tracts (example see Figure $7 \mathrm{~b}$ ) and in $30 \%$ of lobectomized animals. All sham operated and light pulsed insects were rhythmic.

\section{(3) Histological examinations.}

Of the 53 flies with severed optic tracts, 28 had complete cuts on both sides. In nine individuals transection of the optic tract was complete and at the proper position on one side, but on the contralateral side the cut was more distal than anticipated. The remaining 16 flies revealed cuts through optic tracts or lobula complexes on one side and incomplete severance of optic tracts contralaterally (compare Figure 5a).

Neural connections between brain and optic lobes were not established in any of the flies examined histologically. In the majority of the operated flies the optic lobes had degenerated as shown by a large number of small vacuoles (Figures 3 and 4). These degenerated regions. usually stained much deeper than the rest of the brain tissue (Figures 3a, 3b, 4a). Areas of degeneration were found to varying extents even in distal parts of the central brain (Figures $3 c, 4 b, 4 d$ ) but never in mushroom bodies. central body and in antennal lobes. Degeneration in the brain was also observed in some flies with cuts performed through the lobula complexes (Figure 3b) and in individuals with incomplete severance of optic tracts (Figure 3c).

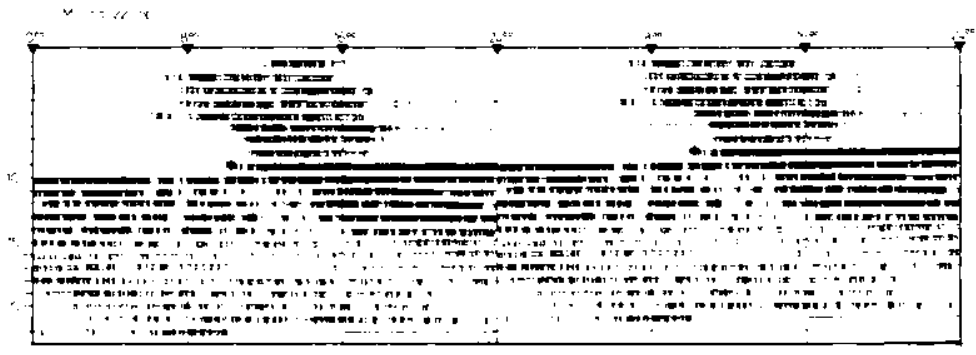

Figure 2. Actogram of an adult Musca domestica under conditions of continuous weak red light. On day 9 both optic lobes were transected (*) as confirmed histologically. The activity rhythm continues with a longer period, increased activity level, and an increased ratio of activity to rest time. 

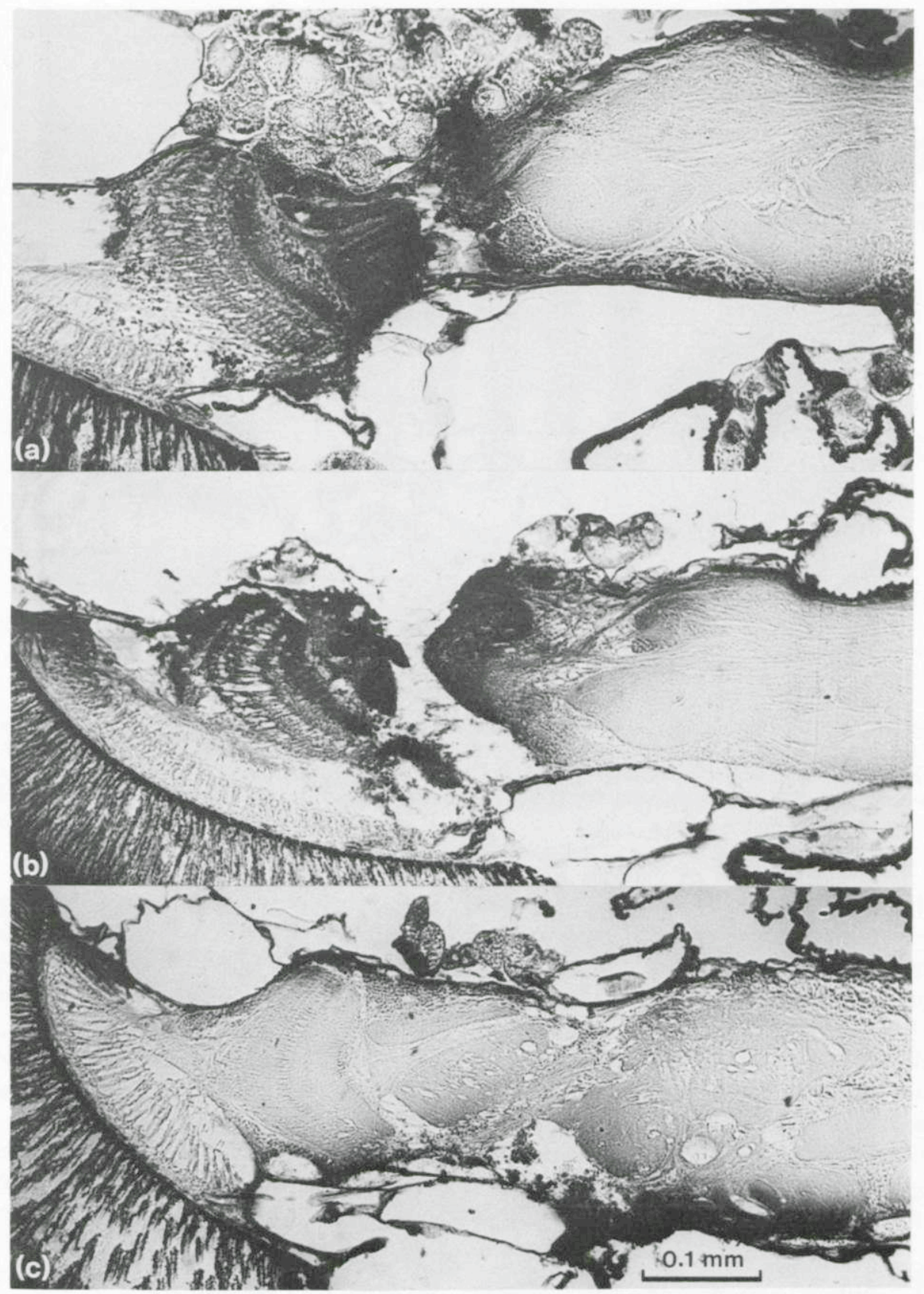

Figure 3. Examples for vacuolized areas (considered to represent degenerated tissue) in the optic lobes (a, b) and the distal part of the central brain (c). (a) Cut between brain and lobula complex with deeply stained optic lobe. Animal remained rhythmic. (b) Cut through lobula complex. Deeply stained optic lobe degenerated on both sides of transection. Animal remained rhythmic. (c) Incomplete cut with degenerations in parts of the optic lobe and distal part of the brain. Animal showed arrhythmic behaviour after operation. 


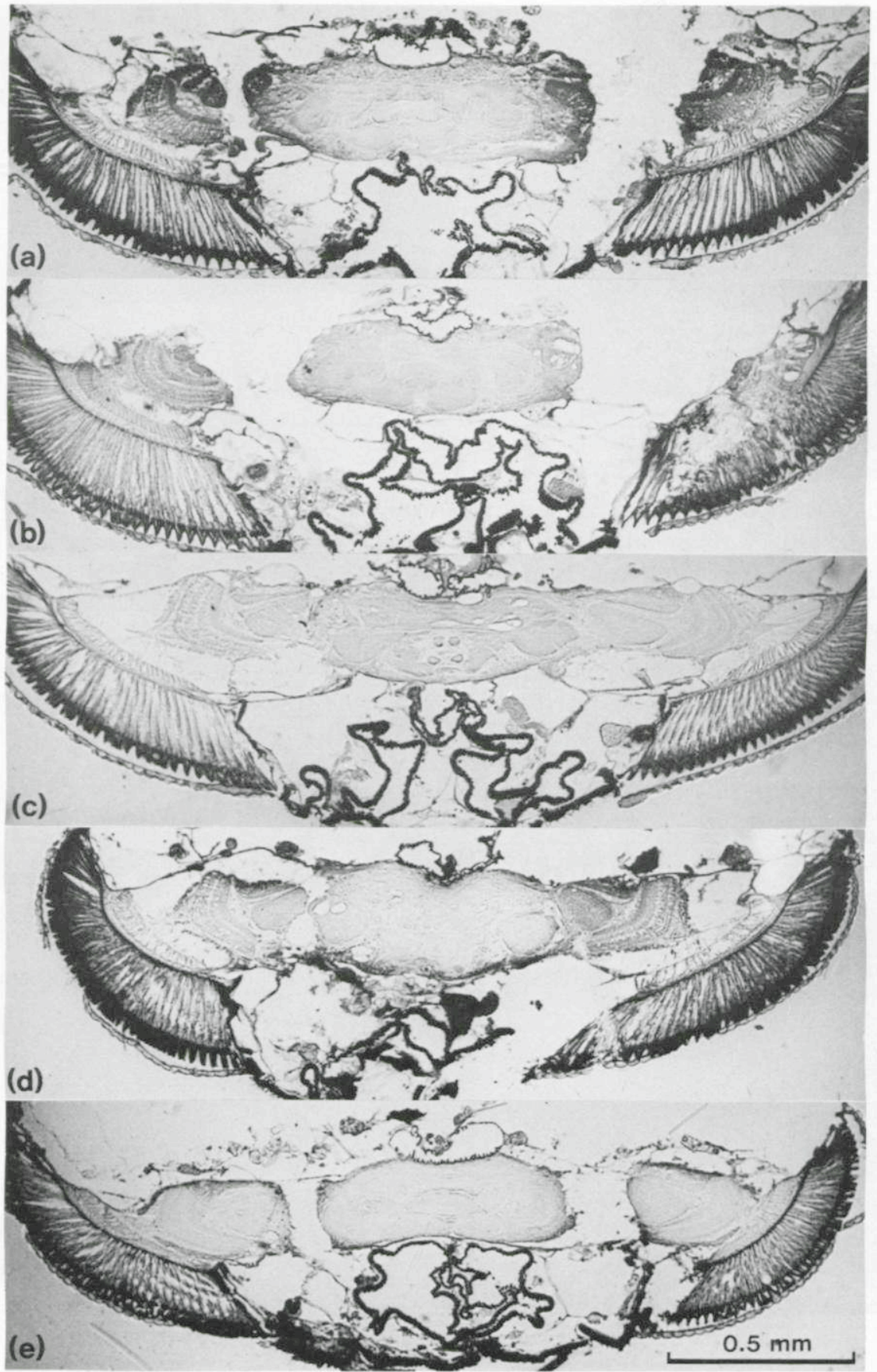

Figure 4. Examples of histological examinations of optic lobes and central brain in Musca domestica illustrating the relationship between location of degenerated tissue and rhythmic behaviour.

(a) Complete cut, optic lobes degenerated, central brain normal appearance. Animal remained rhythmic.

(b) Lobectomy. Parts of the optic lobe still present, but degenerated. No vacuolized areas in brain. Animal remained rhythmic.

(c) A few large vacuoles close to the great commissure. Animal remained rhythmic.

(d) Complete cuts on both sides, optic lobes degenerated, extensive degenerated areas in ventrolateral and posterior protocerebrum. Postoperative behaviour arrhythmic.

(e) Control fly. Staining and fixation immediately after the cut. No vacuolized areas neither in the brain nor in the optic lobes.

\footnotetext{
ch Control fy. Staining and fixation immediately after the cut. No vacuolized areas neither in the brain nor in the optic lobes.
} 


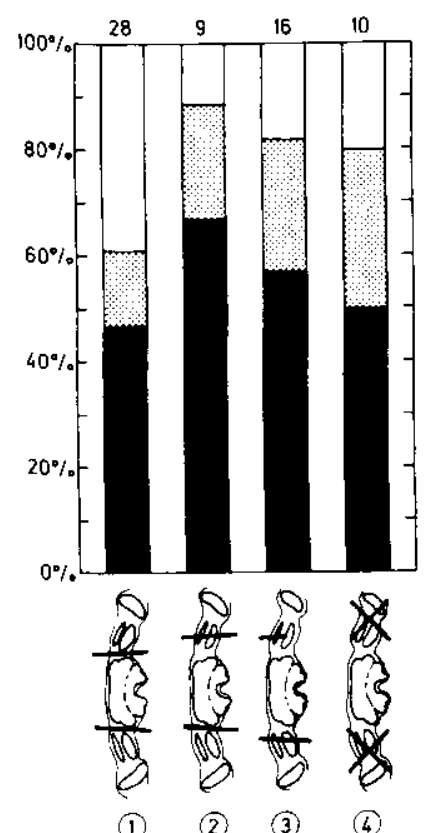

(1) (2) (3) (4)

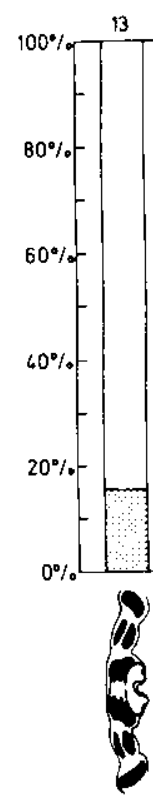

(1)

a
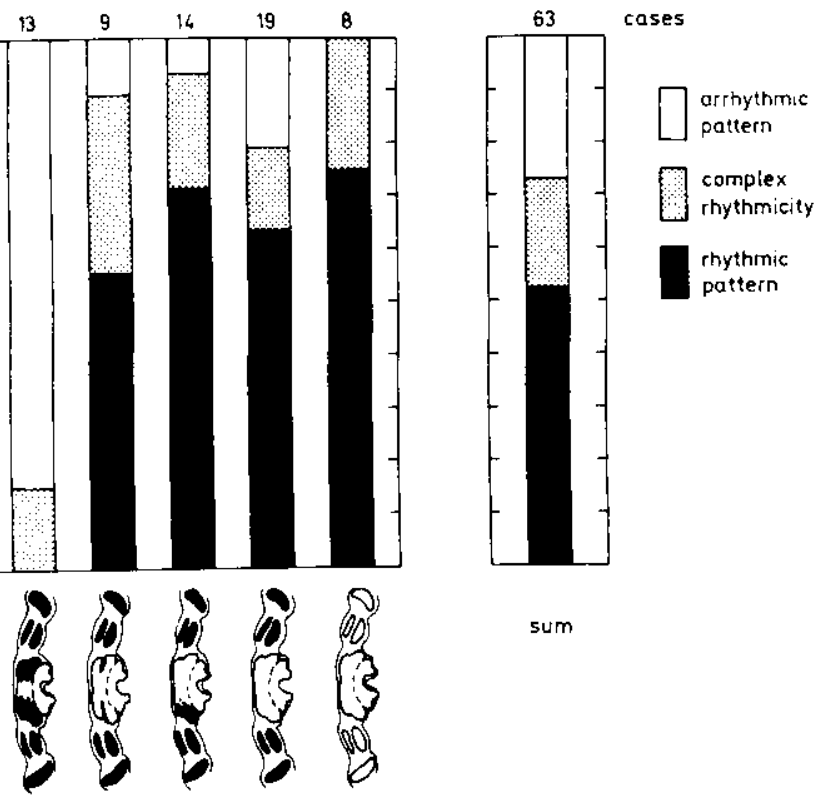

Figure 5. (a) Quantitative relationship between location of optic tract severance (shown below the graph, group 1 to 3 cuts, 4 lobectomy) and kind of locomotor pattern (as explained on the right margin). Number of cases at the top of graph, results in percentage of cases. (b) Quantitative relationship between degree of degeneration (shown below the graph, black areas representing tissue with vacuoles) and kind of locomotor pattern (as explained on the right margin). Last histogram shows sum of (b).

Among lobectomized animals, histological examination revealed that in no case ablation was complete. Small portions of the lamina and sometimes fragments of the lobula complex remained. However, these fragments had no connections with the brain and were completely disorganized (Figure 4b).

(4) Correlation between postoperative rhythms and histological findings.

After recording the locomotor activity of operated flies their brains were carefully examined histologically in order to establish the position of the cut and the degree of degeneration in the brain. No correlation was found between the position of optic tract severance and the percentage of flies exhibiting rhythmicity, complex rhythmicity or arrhythmicity (Figure 5a). However, these types of postoperative behaviour were correlated with the presence of vacuolized regions in the central brain (Figure 5b): The percentage of arrhythmic flies was significantly higher in group 1 (Figure $5 b)$ with vacuolized regions in both lateral parts of the brain as compared to the other groups. All flies lacking vacuolized regions (group 5, Figure $5 b)$ were rhythmic. Of all arrhythmic flies $65 \%$ showed such degenerations within the brain. $30 \%$ of the arrhythmic flies had no such degenerated areas within the brain. However, these insects were extremely hyperactive (Figure $7 \mathrm{~d}$ ) which makes the detection of rhythmicity in the actograms difficult.

Some flies with rhythmic postoperative behaviour had also vacuolized areas within the brain. However, these were minor as compared to those of arrhythmic flies and never occurred simultaneously on both sides of the brain within ventrolateral and posterior parts of the protocerebrum. Vacuolized areas were mainly located between these two parts of the brain in the rhythmic flies (Figure $4 \mathrm{c}$ ). In the case of flies 
exhibiting complex postoperative rhythmicity no correlation was found between degenerations and its localization within the brain.

\section{(5) Characteristic of postoperative rhythms}

After severing the optic tracts 25 of the rhythmic flies were recorded long enough to allow the precise analysis of their postoperative rhythms. This included determination and comparison of preoperative and postoperative period length (Table 3 ) and phase of the rhythm. Almost all flies lengthened period after the operation. This was, however, also found in flies which were sham operated or illuminated only.

Table 3. Changes in period length of locomotor activity rhythm in Musca domestica after bilateral severance of optic tracts, bilobectomy, sham operation and light pulse treatment only. Period length determined with periodogram analysis, peaks above $95 \%$ confidence limit. Mean and standard errors given

\begin{tabular}{lcccc}
\hline & $\begin{array}{c}\text { Number } \\
\text { of cases }\end{array}$ & $\begin{array}{c}\text { Before treat- } \\
\text { ment (hr) }\end{array}$ & $\begin{array}{c}\text { After treat- } \\
\text { ment }(\mathrm{hr})\end{array}$ & $\begin{array}{c}\text { Difference } \\
\Delta \tau(\mathrm{hr})\end{array}$ \\
\hline $\begin{array}{l}\text { Optic tracts } \\
\text { severed }\end{array}$ & 20 & $24.9 \pm 0.16$ & $25.6 \pm 0.14$ & $0.66 \pm 0.11$ \\
Lobectomy & 3 & $24.8 \pm 0.48$ & $26.0 \pm 0.45$ & $1.23 \pm 0.07$ \\
Sham & 20 & $25.3 \pm 0.18$ & $25.6 \pm 0.10$ & $0.31 \pm 0.13$ \\
Light pulse & 15 & $25.0 \pm 0.23$ & $25.3 \pm 0.23$ & $0.32 \pm 0.05$ \\
\hline
\end{tabular}

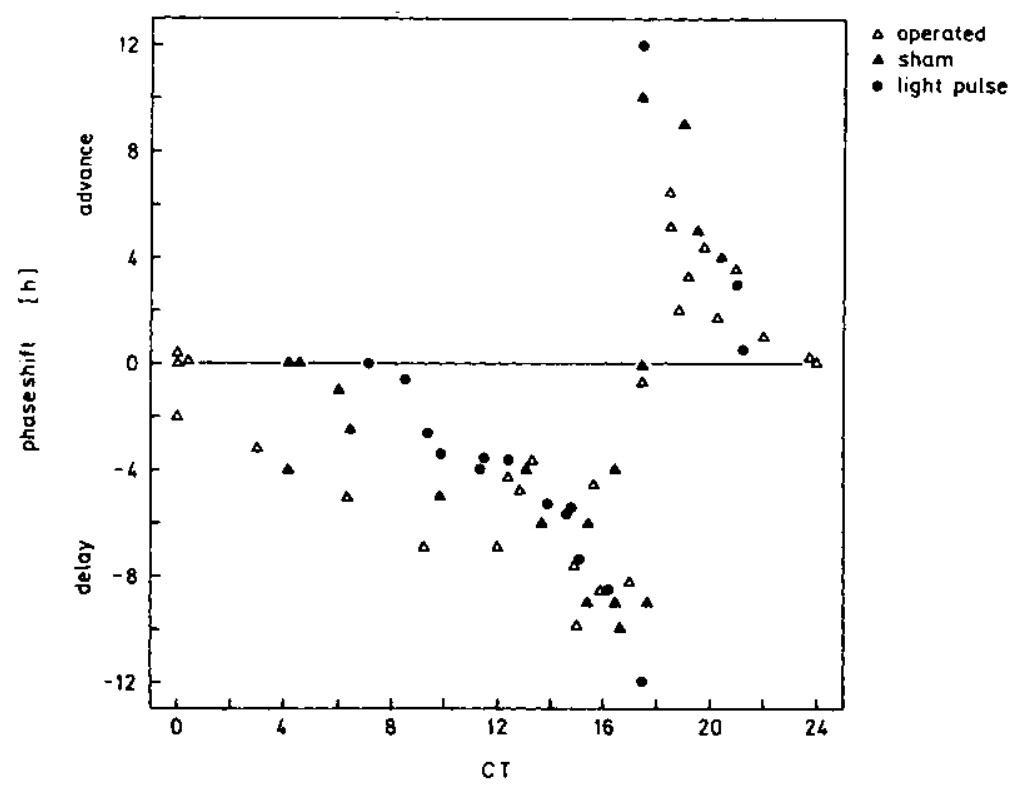

Figure 6. Phase response after 5 min white light $\left(6 \times 10^{4} 1 x\right)$ illumination at different circadian times (CT 0 is onset of activity). Operated (optic tract severed or lobectomy), sham operated and unoperated cases in different symbols. 
nal Circadian Activity Rhythm of the House Fly
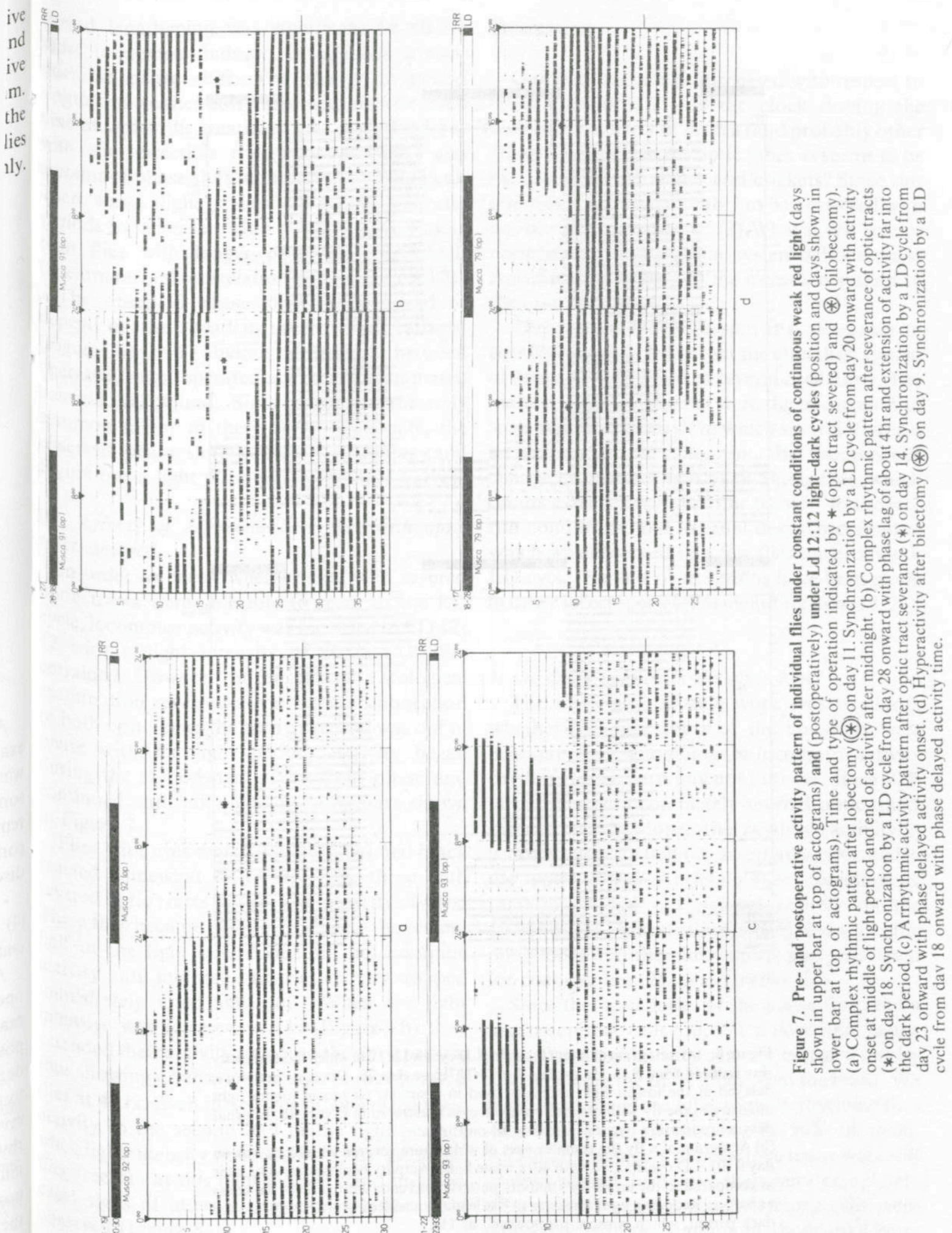

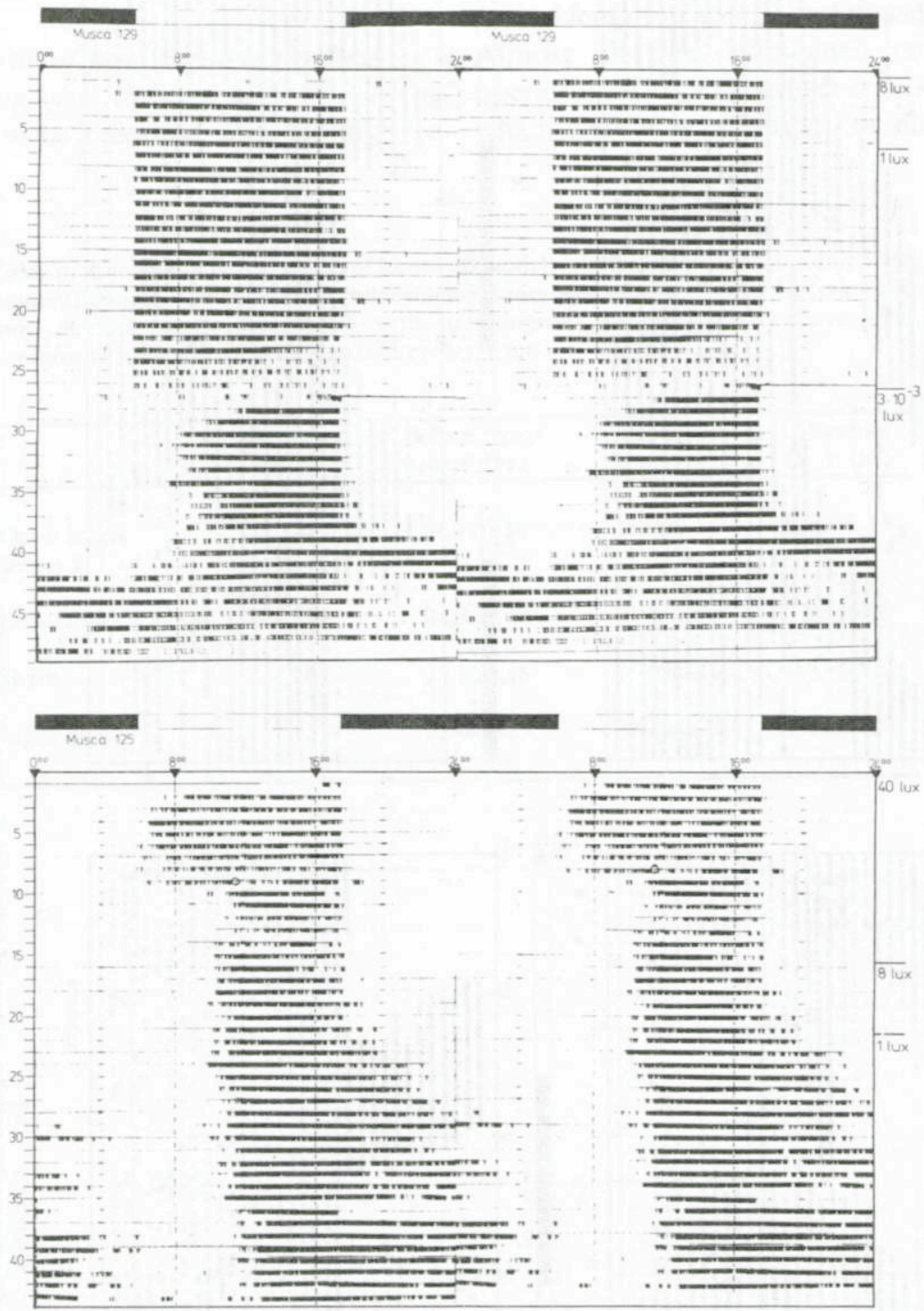

Figure 8. (a) Actogram of intact fly under LD cycles $12: 12$ in which light intensity was reduced from 8 to $1 \mathrm{~lx}$ on day 7 to $3 \times 10^{-3} \mathrm{~lx}$ on day 26 . Onset of activity is delayed at the lowest light intensity used in a similar way as at higher light intensity in the fly with covered complex eyes illustrated in Figure 8b. Internal desynchronization occurs from day 38 onward.

(b) Actogram of a fly the complex eyes of which were covered with black wax on day $9(0)$. LD cycle 12:12 with $40 \mathrm{~lx}$ white light. Activity begins several hr later as compared to the intact fly's activity pattern, and ends with onset of darkness as before. Reducing light intensity to $8 \mathrm{~lx}$ leads to a widening of activity into the dark period, which is more pronounced at $11 \mathrm{x}$.

Period

Most $\operatorname{tr}$

siderabls

length $\mathrm{x}$

time at

with th

amount

There $u$

periods

than $\mathrm{fl}$

Spearn

The

depend

(Figure

Operate

animals

commo

observe

Figure

(6) Ef

tracts s

In 0

optic t

cycle, 1

12 wit

entrain

examir

of botl

phase

during

contin

in Fig!

Flie:

reacter

severe

These

half o

activit

immer

intens

extenc

thus s'

flies a

activit

when

flies $\mathrm{f}$

cases

obser 
Period lengthening was significant ( $a=0.01$ ). Most treatments induced in addition a considerable phase shift. The change in period length was neither correlated with the circadian time at which the treatment was performed nor with the direction (delay or advance) and amount of phase shift induced by the treatment. There was a slight tendency in flies with shorter periods to increase period postoperatively more than flies with longer postoperative periods (Spearman rank correlation coefficient $r_{s}=0.5$ ).

The observed phase shifts were found to depend on the circadian time of the treatment (Figure 6). No obvious difference between operated, sham operated and solely illuminated animals was found. Since light was the only common factor in these three treatments, the observed phase shifts are light responses (and Figure 6 is a light pulse phase response curve).

(6) Effects of $L D$ conditions flies with optic tracts severed.

In order to check whether flies with severed - optic tracts were still able to react to the LD cycle, locomotor activity was recorded in LD 12 : 12 with a light intensity of $40 \mathrm{~lx}$. All flies entrained. However, in flies whose histological examination revealed a complete disconnection of both optic lobes the activity period was out of phase with the light period. Activity began during the second part of the light phase and continued until midnight. Examples are shown in Figure 7.

Flies with eyes which had been painted black reacted somewhat differently than those with Severed optic tracts at the light intensity of $401 \mathrm{x}$. These flies became also active during the second half of the light period but did not continue activity until midnight. Instead activity stopped immediately after lights off. When the light intensity was lowered to 1 lx (Figure 8b) they extended their activity time into the dark period, thus showing a behaviour similar to the operated flies at $40 \mathrm{~lx}$. Even intact flies often shifted their activity to the second half of the light period, when light intensity was reduced to $10^{-3} \mathrm{~lx}$. Some flies free ran at this light intensity and in a few cases signs of internal desynchronization were observed (Figure 8a).

\section{Discussion}

Our findings can be discussed with respect to three questions: (1) Is the clock driving the locomotor activity of $\mathrm{Musca}$ (and probably other Diptera) located in the optic lobes as seems to be the case in cockroaches and crickets? Since this question has most probably to be answered with no, the next question is: (2) What is the role of optic lobes in the circadian system of house flies? And finally: (3) How is the circadian system of flies synchronized?

Throughout the discussion it is necessary to consider the possibility that the circadian system of Musca is composed of several oscillators. The main arguments for this were the occurrence of 'complex rhythmicity', in which several rhythms were found in an actogram, the spontaneous change in period length of rhythmic flies, which occurs often some days after transfer to the free run conditions, and internal desynchronization which was observed in a few flies under a weak LD cycle. Similar observations have been made in other insect species (23) including flies $(17,24)$.

\section{Is the clock located in the optic lobe?}

The main goal of this work was to clarify whether the optic lobes of the house fly were necessary for maintaining the locomotor activity rhythm. This is certainly not the case. Since $50 \%$ of the flies with completely severed optic tracts showed clear postoperative rhythmic activity, it is rather unlikely that the pacemaker is located in the optic lobes. At least, the expression of rhythmicity can not depend entirely on the existence of neural connections between the optic lobes and the midbrain as was postulated for cockroaches (25) and Gryllus (8).

Since the optic lobes of the house fly contain neurosecretory cells $(26,27)$, a hormonal factor controlling rhythmicity can not be ruled out by disconnecting the optic lobes from the brain. We performed therefore lobectomy in rhythmic flies and still found rhythmicity in $50 \%$ of them. Although fragments of optic lobe tissue were still present after lobectomy, they were completely disorganized and no neurosecretory cells were detectable with the staining methods used here. 
The same was found in most of the flies with optic tracts severed.

It seems therefore that the pacemaker is located in the midbrain of the fly. In order to localize it more precisely we tried to correlate the postoperative behaviour of the flies with the histological appearance of their brains. We found that most arrhythmic flies had vacuolized areas in the brain after severance of both optic tracts. However, the exact location for the pacemaker could not be identified, because degenerations were located in different parts of the brain and varied from fly to fly. The observed degenerations within the brain might be due rather to destruction of the tracheal system supplying oxygen than to the severance of the optic tracts. This could explain the fact that in some flies with incomplete optic tract severance and sometimes in sham operated flies vacuolized areas were found in the optic lobes and in the midbrain. Damage of tracheal system might also be the main factor preventing regeneration of the severed optic tracts of flies. Regeneration of the connection between the optic lobes and the brain has been observed in the case of cockroaches (25) and Gryllus (8).

To assertain that the fixing and staining procedures were not the cause of the observed vacuolisation, the same procedures were applied to brains immediately after optic tract severance. Neither in the brain nor in the optic lobes could any degeneration areas be detected in these cases (fig. 4e). Our results thus suggest that the optic lobes of the house fly are not necessary for maintaining the circadian rhythm of locomotor activity. This is in accordance with findings in the fruit fly in which mutations reducing the optic lobes in size to about $5 \%$ did not abolish the locomotor activity rhythm (28) and with recent findings of Kasai and Chiba (18) in Culexpipiens.

Role of the optic lobes in the circadian system of house flies

Although the optic lobes of house flies are not the site of the clock, it seems that they play a role in the oscillatory system of this insect. This conclusion is based on the postoperative behaviour of lobectomized flies and flies with optic tracts severed showing three major changes: (1) appearance of complex rhythmicity. (2) lengthening of the period of the rhythm and (3) increase in activity level.

Appearance of complex rhythmicity: As. mentioned already, a certain percentage of the operated flies exhibit a complex activity patter composed of different rhythms with differing period lengths (periodogram analysis). In Drosophila mutants with reduced optic lobes tht occurrence of such complex activity patterns is more frequent than in the wild strain $(17,18)$. However, in Drosophila complex rhythmicit! usually consisted of two components with period lengths of about 22 and $25 \mathrm{hr}$. There was nol. much interindividual variation in these periods. House flies with two or four components. however, lacked the regularity of period length of the Drosophila mutants. Nevertheless the occurrence of complex rhythmicities doe: support the view that more than one oscillator is involved in the expression of the overt rhythmol locomotor activity and that the optic lobes play role in the mutual coupling (17).

Lengthening of the period of the rhythri period lengthening was observed in all groups postoperatively. The period lengthening in lobectomized flies was almost twice as largt $(+1.23 \mathrm{hr})$ as in animals with severed optic tract $(+0.66 \mathrm{hr})$, and this was twice as large as aftel sham operation $(+0.31 \mathrm{hr})$. There seem therefore to be a slight correlation between the degree of damage to the optic lobes and periol lengthening. However, these differences were not statistically significant among each other. Ever the light pulsed control flies lengthened theif period $(+0.32 \mathrm{hr})$.

Aschoff (23) reported period changes in vertebrates after light pulses. Period was lengthened by light pulses causing phase delays. and shortened by light pulses causing phas advances. In the case of the house fly, period wa lengthened by both phase advancing an delaying light pulses. Page et al. (30) foun period lengthening for the locomotor activit? rhythm in cockroaches after removal of or optic lobe. They explained this finding b! assuming that circadian pacemakers wert located in both lobulae and mutually coupled. the coupled state the period length of the systert 
was thought to be shorter as compared to each single oscillator. The optic lobes might play also a role with respect to the coupling of oscillators located in the central brain as has been discussed in Drosophila mutants with strongly reduced optic lobes in regard to the lengthened period of locomotor activity rhythms. Whether and in which way optic lobes influence the coupling of oscillators in Musca need further studies.

Increase in activity level: an increase in activity level has been observed in several insect species after different surgical treatment $(3,31,32)$. In the case of crickets $(32,33)$ an increased level of locomotor activity seems to be due to a disturbance in the functioning of neurosecretory cells. Neurosecretion liberated rhythmically from the brain has an inhibitory effect on the locomotor activity of the insect $(33,12)$. It is possible, that in house flies severance of the optic tracts causes a disturbance in the normal function of the neurosecretory cells. This possibility is now under investigation.

\section{Photoreception and entrainment}

Contrary to the situation in Drosophila pseudoobscura (34) the circadian rhythm of the locomotor activity of house flies seems to be quite sensitive to light. Substantial phase shifts of up to 12 hrwere found after a single exposure to 5 min of white light. A tentative phase response curve is shown in Figure 6, but needs further work. Both, phase advances and delays were found.

Under LD conditions flies are entrained and active during the light period. Some flies show a bimodal activity as already observed by Parker (35) and, for Drosophila pseudoobscura, by Mack (34). Painting the complex eyes black suppresses activity in the first part of the light period (Figure $8 b)$ and in cases of bimodality the first activity peak is missing. In any case flies with covered compound eyes as well as operated flies were still synchronized by LD cycles suggesting that the compound eyes are not the decisive photoreceptors for the oscillatory system controlling activity. However, the compound eyes seem to be responsible for the occurrence of the activity peak immediately after lights on.

Operated flies began activity at about midday as did flies with eyes painted black, but extended activity into the dark period. Their activity time was therefore much longer than that of flies with black painted complex eyes. This increase in activity time might have been caused by a weakened coupling of normally more tightly coupled oscillators. A tight coupling results in a distinct and narrow band of activity. If the coupling were loosened the activity pattern would lose its distinct onset and end and widen. If coupling were further decreased, several rhythms might occur. Finally, with even less coupling, arrhythmicity would occur. A widening of the activity pattern was observed in some of the flies with covered eyes if light intensity was reduced (Figure $8 \mathrm{~b}$ ). Under these circumstances, the Zeitgeber is apparently not strong enough to synchronize all components of the oscillatory system. Some flies with covered complex eyes and even some normal flies under very low light intensities showed internal desynchronization (Figure 8a).

Leaving speculation aside, our most important result is the fact that even arrhythmic and hyperactive flies can be synchronized to LD cycles. This is probably not a direct response to light, but indicates the participation of an endogenous clock. Otherwise we would expect activity to begin immediately after light on. If this reasoning is correct, even overt arrythmic flies would possess a clock. However, its amplitude was too weak to maintain a clear rhythm under continuous RR. Or, alternatively, the internal coupling between the oscillators was not strong enough to bring about a distinct activity pattern in RR or DD. An external Zeitgeber, however, was able to synchronize the oscillators.

The photoreceptor for synchronization of blind flies is not yet known. We are planning to determine the action spectrum of phase shifting light pulses in blind house flies and blind mutants of Drosophila.

Acknowledgements-This work was supported in part by scientific programme R-III-14 coordinated by the JagielIonian University, Cracow. Poland. We are thankful to Mrs. Wigand and Dr. Hausen for supply with flies, and Prof. Bunning and Dr. Dittami for reading the manuscript. 


\section{References}

1. Page T. L. Localization of circadian pacemakers in insects. In: Follet B. K. and Follet D. E., eds., Biological Clocks in Seasonal Reproductive Cicles. John Wright, Bristol, pp. 113-124, 1981.

2. Rusak B. and Zucker I. Neuroregulation of circadian rhythms. Physiol Rev 59, 449-526, 1979.

3. Nishiitsutsuji-Uwo J. and Pittendrigh C. S. Central nervous system control of circadian rhythmicity in the cockroach-III. The optic lobes, locus of the driving oscillations? Z Verg/ Physiol 58, 14-46, 1968.

4. Roberts S. K. Circadian rhythms in cockroaches. Effects of optic lobe lesions. J. Comp Physio/ 88, $21-30,1974$.

5. Page T. L. Interaction between bilaterally paired components of the cockroach circadian system. J Comp Phy'siol 124 . $225-236,1978$.

6. Page T. L. Transplantation of the cockroach circadian pacemaker. Science 216, 73-75, 1982.

7. Sokolove P. C. and Loher W. Role of eyes, optic lobes and pars intercerebralis in locomotory and stridulator? circadian rhythms of Teleogryllus commodus. J Insect Physiol 121, 785-799, 1975.

8. Tomioka K. and Chiba Y. Effects of nymphal stage optic nerve severance or optic lobe removal on the circadian locomotor rhythm of the cricket, Gryllus bimaculatus. Zool Sci 1, 375-382, 1984.

9. Balkenohl M. and Weber F. Sind auch bei holometabolen Insekten circadiane Schrittmacher der Aktivität in den optischen Ganglien tokalisiert? Mitl Disch Ges Allg Angew Ent 3, 223-227, 1981.

10. Cymborowski B. and Brady J. Insect circadian thythms transmitted by parabiosis-a reexamination. Nature 236 . $221-222,1972$.

11. Cymborowski B. and Dutkowski A. Circadian changes in protein synthesis in the neurosecretory cells of the central nervous system of Acheta domesticus. J. Insect Physiol 46, 341-348, 1970.

12. Cymborowski B. Daily changes in synthesis and accumulation of neurosecretion in the brain of the house cricket. Interdiscipl Cycle Res 14, 111-116, 1983.

13. Handler A. M. and Konopka R. J. Transplantation of a circadian pacemaker in Drosophila. Nature 279. 236-238, 1979.

14. Konopka R. J. and Wells S. Drosophila clock mutations affect the morphology of a brain neurosecretory cell group. $J$ Neurobiol $11,411-415,1980$.

15. Truman J. W. Physiology of insect thythms. II. The silkmoth brain as the location of the biological clock controlling eclosion. J Comp Physiol 81, 99-114, 1972.

16. Truman J. W. Physiology of insect rhythms-IV. Role of the brain in the regulation of the flight rhythm of the giant silkmoth. J Comp Phy'siol 95, 281-296, 1974.

17. Helfrich C. and Engelmann W. Circadian rhythm of the locomotor activity in Drosophila melanogaster and its mutants "sine oculis" and "small optic lobes". Physiol Entomol 8, 257-272, 1983.

18. Kasai M. and Chiba Y. The mosquito clock is not in the optic lobes. 10th Intern. Congress of Biometeorology, Tokyd. 1984.

19. Engelmann W. and Mack J. Different oscillators control the circadian rhythm of eclosion and activity in Drosophila.' Comp Physiol 124, 225-236, 1978.

20. Martin W. and Brinkmann K. A computer program system for the analysis of equispaced time series. $J$ Interdiscipl $C y c l t$ Res 7, 251-258, 1976.

21. Siegel S. Nonparametric Statistics for the Behavioral Sciences. McGraw-Hill, New York. 1956.

22. Case R. Differentiation of the effects of $\mathrm{pH}$ and $\mathrm{CO}_{2}$ on the spiracular function of insects. $J$ Cell Comp Physiol 49 . $103-113,1957$.

23. Christensen N. D. and Lewis R. D. The circadian locomotor thythm of Hemideina thoracica (Orthoptera; Stenopeimat dae): the circadian clock as a population of interacting oscillators. Physiol Entomol 7, 1-13, 1982.

24. Waddel B. M.Sc. dissertation, University of Auckland, New Zealand, 1983.

25. Page T. L. Regeneration of the optic tracts and circadian pacemaker activity in the cockroach Leucophaea maderat. J Comp Physiol 152, 231-240. 1983.

26. Köpf H. Über Neurosekretion bei Drosophila-I. Zur Topographie und Morphologie neurosekretorischer Zentren bel der Imago von Drosophila. Biol Zentralblatt 76, 28-42, 1957.

27. Thomsen $M$. The neurosecretory system of the adult Calliphora erythrocephala II. Histology of the neurosecretory cells of the brain and some related structures. Z Zellforsch Mikrosk Anat 67, 697-717, 1965.

28. Helfrich C. Locomotor activity rhythm of the Drosophila mutants "optomotor blind", "lobula plateless", "minibrain". "minibrain/sine oculis" and small optic lobes/sine oculis". In preparation

29. Aschoff J. Circadian rhythms: Influences of internal and external factors on the period measured in constant conditions. $Z$ Tierpsychol 49, 225-249, 1979.

30. Page T. L.. Caldarola P. C. and Pittendrigh C. S. Mutual entrainment of bilaterally distributed circadian pacemakers. Proc Natl Acad Sci USA 74, 1277-1281, 1977.

31. Brady $\mathbf{J}$. Control of the circadian rhythm of activity in the cockroach-II. Role of the sub-oesophageal ganglion and ventral nerve cord. $J$ Exp Biol 47, 165-178, 1967.

32. Cymborowski B. Control of the circadian rhythm of locomotor activity in the house cricket. $J$ Insect Physiol 19. $1423-1440,1973$.

33. Cymborowski B. Transplantation of circadian pacemaker in the house cricket Acheta domesticus L. Interdiscip/ CyClt Res 121, 133-140, 1981 .

34. Mack J. Das Multioscillatorsystem von Drosophila melanogaster. Doctoral dissertation, Universität Tübingen. Germany, 1980.

35. Parker A. H. Studies on the diurnal rhythms of the house fly, Musca domestica L., in a dry tropical environment. Acto Trop 19. 97-119, 1962.

36. Straussfeld N. J. Atlas of an Insect Brain. Springer, Berlin. 1976. 